[white paper]

Diamond Open Access

\title{
A unidimensional windmill in the plane with varying pivot points
}

\author{
Open Mathematics Collaboration*†
}

August 27, 2020

\begin{abstract}
We show that we can choose a point in the plane such that the resulting windmill process with varying pivot uses each point of the plane infinitely many times.
\end{abstract}

keywords: international mathematical olympiad, imo, logic, set theory

The most updated version of this paper is available at https://osf.io/mwkaf/download

\section{How to co-author/co-sign this paper?}

1. Send us a complete review report and answer the following questions.

2. Which discussions would you add to this paper?

3. Did you find any mistakes or inconsistent arguments? Give a detailed justification.

*All authors with their affiliations appear at the end of this paper.

†Corresponding author: mplobo@uft.edu.br | Join the Open Mathematics Collaboration 


\section{Introduction}

4. We discuss the solution of Problem 2 of the International Mathematical Olympiad (IMO, 2011) [1,2].

\section{Definitions}

5. $\mathcal{S}=$ a finite set of at least two points in the plane

6. No three points of $\mathcal{S}$ are collinear.

7. windmill $=$ the rotation process that starts with a line $\ell$ going through a single pivot point $P \in \mathcal{S}$

8. $\ell$ rotates clockwise about $P$ until the first time that $\ell$ meets some $Q \in \mathcal{S}$

9. $Q=$ new pivot

10. $\ell$ rotates clockwise about $Q$ until it next meets a point in $\mathcal{S}$

11. This process continues indefinitely.

\section{Theorem}

12. We can choose $P \in \mathcal{S}$ and $\ell$ going through $P$ such that the resulting windmill uses each point of $\mathcal{S}$ as a pivot infinitely many times [1].

\section{Proof}

13. Let $\ell$ have an orientation (Fig. 1).

14. $\ell$ divides the plane into the grey and white sides.

15. Start with $\ell$ going through a point $O$ such that all points in $\mathcal{S}$ are in the white side. 

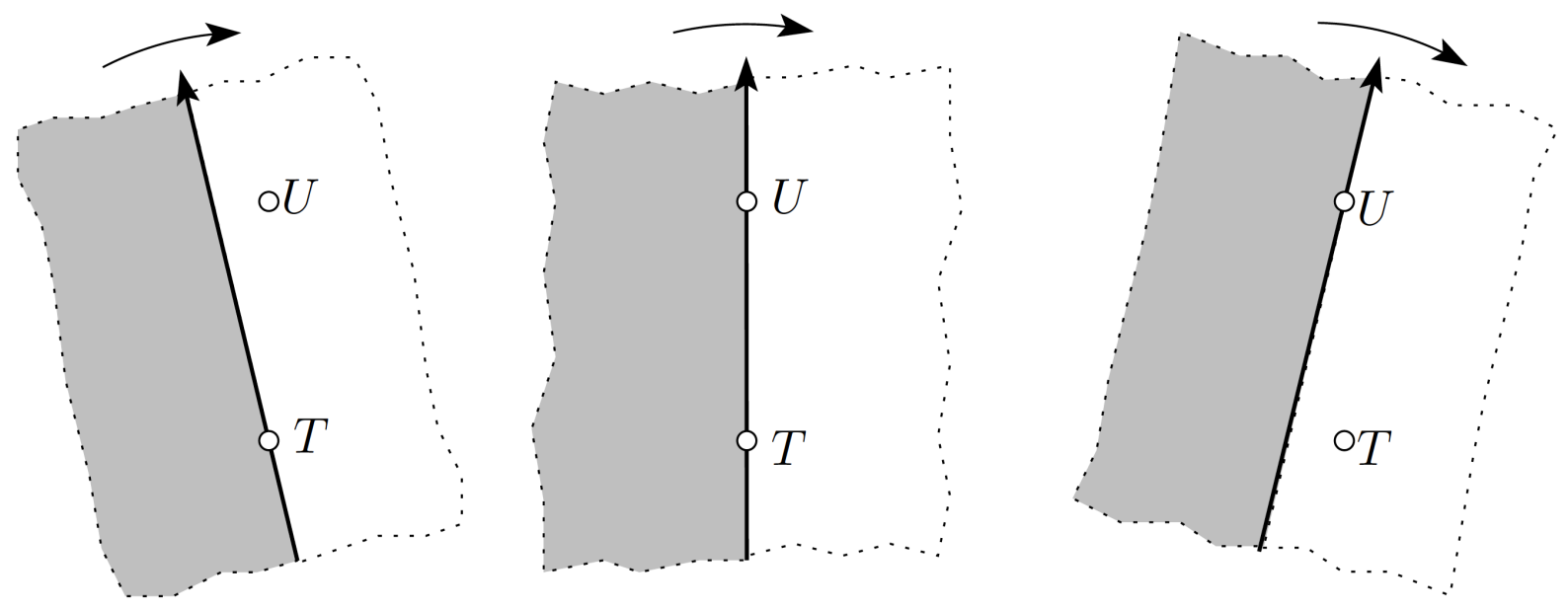

Figure 1: Two arbitrary points and the oriented rotating line are shown [1].

16. Fix the pivot in $O$.

17. Since (6), as $\ell$ moves clockwise (with the pivot fixed at $O$ ), it passes through one point at a time.

18. Move $\ell$ until there are

(a) $n$ points in each side if $|\mathcal{S}|=2 n+1$.

(b) $n-1$ points in the grey side and $n$ in the white if $|\mathcal{S}|=2 n$.

19. Now, start the windmill process as described in (7-11).

20. Note that whenever the pivot changes from $T$ to $U$ (Fig. 1), after the change, $T$ is on the same side as $U$ was before.

21. The number of elements of $\mathcal{S}$ on the grey side and the number of those on the white side remain the same throughout the whole process (except for those moments when the line contains two points).

22. Let the configuration in (18) be the starting line.

23. During the rotation, due to (20), each point from the grey side becomes white and vice versa. 
24. Therefore, after having made a rotation of $180^{\circ}$, the windmill should have passed through all the points.

\section{Open Invitation}

Review, add content, and co-author this paper [3,4]. Join the Open Mathematics Collaboration (https://bit.ly/ojmp-slack). Send your contribution to mplobo@uft.edu.br.

\section{Open Science}

The latex file for this paper together with other supplementary files are available [5].

\section{Ethical conduct of research}

This original work was pre-registered under the OSF Preprints [6], please cite it accordingly [7]. This will ensure that researches are conducted with integrity and intellectual honesty at all times and by all means.

\section{Acknowledgement}

+ Center for Open Science https://cos.io

+ Open Science Framework https://osf.io 


\section{References}

[1] IMO. International Mathematical Olympiad (2011).

https://www.imo-official.org/

[2] 3Blue1Brown (2019). https://youtu.be/M64HUIJFTZM

[3] Lobo, Matheus P. "Microarticles." OSF Preprints, 28 Oct. 2019. https://doi.org/10.31219/osf.io/ejrct

[4] Lobo, Matheus P. "Simple Guidelines for Authors: Open Journal of Mathematics and Physics." OSF Preprints, 15 Nov. 2019. https://doi.org/10.31219/osf.io/fk836

[5] Lobo, Matheus P. "Open Journal of Mathematics and Physics (OJMP)." OSF, 21 Apr. 2020. https://doi.org/10.17605/osf.io/6hzyp

[6] COS. Open Science Framework. https://osf.io

[7] Lobo, Matheus P. "A Unidimensional Windmill in the Plane with Varying Pivot Points." OSF Preprints, 26 Aug. 2020. https://doi.org/10.31219/osf.io/mwkaf

\section{The Open Mathematics Collaboration}

Matheus Pereira Lobo (lead author, mplobo@uft.edu.br) $)^{1,2}$ https://orcid.org/0000-0003-4554-1372

Hans Müller Silva Oliveira ${ }^{1,3}$

https://orcid.org/0000-0002-7137-9789

${ }^{1}$ Federal University of Tocantins (Brazil)

${ }^{2}$ Universidade Aberta (UAb, Portugal)

${ }^{3}$ Colégio Invictos (Araguaína, Tocantins, Brasil) 